\title{
UPPER AND LOWER BOUNDS OF THE BASIS NUMBER OF KRONECKER PRODUCT OF A WHEEL WITH A PATH AND A CYCLE
}

\author{
*Ghassan T. Marougi Ayhan A. Khalil \\ *Dept. Math./College of Computers \& Mathematics Sciences/ \\ Mosul University
}

Received

27/7/2006
Accepted

3/10/2006

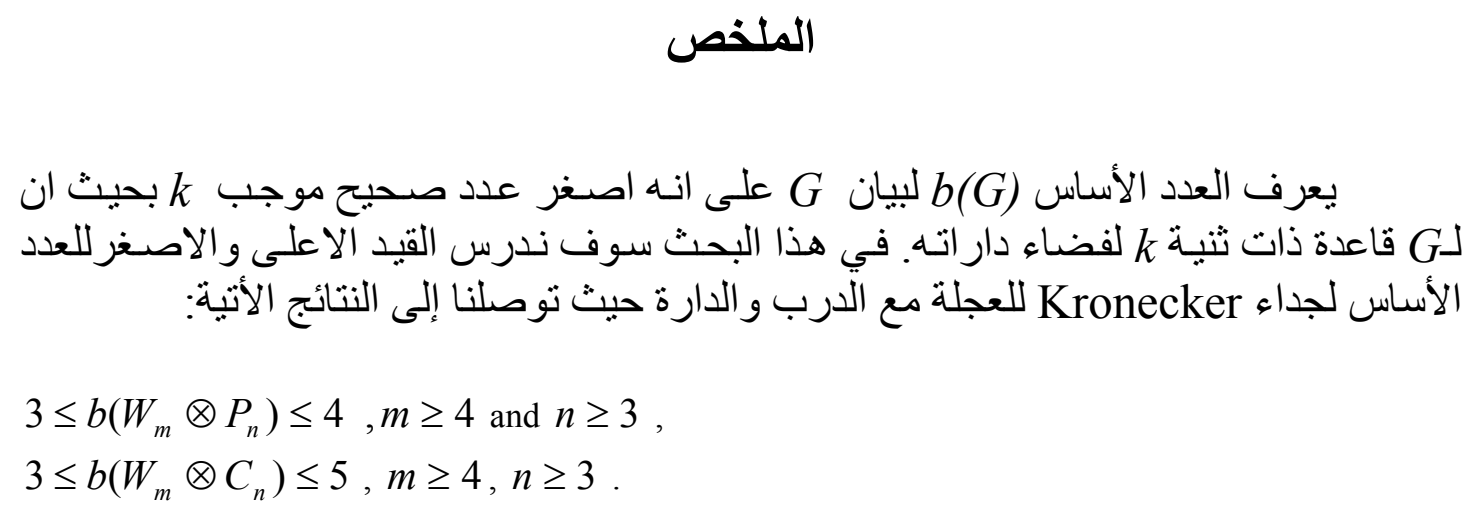

\section{ABSTRACT}

The basis number, $b(G)$, of a graph $G$ is defined to be the smallest positive integer $k$ such that $G$ has a $k$-fold basis for its cycle space. We investigate upper and lower bounds of the basis number of Kronecker product of a wheel with a path and a cycle. It is proved that

$3 \leq b\left(W_{m} \otimes P_{n}\right) \leq 4, m \geq 4$ and $n \geq 3$,

and

$$
3 \leq b\left(W_{m} \otimes C_{n}\right) \leq 5, m \geq 4, n \geq 3 .
$$




\section{INTRODUCTION.}

Throughout this paper, we consider only finite, undirected and simple graphs. Our terminology and notations will be standard except as indicated. For undefined terms, see [3] .

Let $G$ be a connected graph, and let $e_{1}, e_{2}, \ldots \ldots, e_{q}$ be an ordering of the edges. Then any subset $S$ of edges corresponds to a $(0,1)$-vector $\left(a_{1}, a_{2, \ldots . .,}\right.$, $a_{q}$ ) in the usual way, with $a_{i}=1$ if $e_{i} \in S$ and $a_{i}=0$ otherwise, for $i=1,2, \ldots . . q$. These vectors form a q-dimensional vector space, denoted by $\left(Z_{2}\right)^{q}$ over the field $Z_{2}$.

The vectors in $\left(Z_{2}\right)^{q}$ which correspond to the cycles in $G$ generate a subspace called the cycle space of $G$, and denoted by $\xi(G)$. It is well known that

$$
\operatorname{dim} \xi(G)=\gamma(G)=q-p+k,
$$

where $\mathrm{p}$ is the number of vertices, $k$ is the number of connected components and $\gamma(G)$ is the cyclomatic number of $G$. A basis for $\xi(G)$ is called h-fold if each edge of $G$ occurs in at most $h$ of the cycles in the basis. The basis number of $G$, denoted by $b(G)$, is the smallest positive integer $h$ such that $\xi(G)$ has an h-fold basis, and such a basis is called a required basis of $G$ and denoted by $B_{r}(G)$. If $B$ is a basis for $\xi(G)$ and e is an edge of $G$, then the fold of $e$ in $B$, denoted by $f_{B}(e)$ is defined to be the number of cycles in $B$ containing $e$.

Definition: Let $G=(V, E)$ be a simple graph with order $n$ and vertex set $V=\left\{p_{1}, p_{2}, \ldots, p_{n}\right\}$. the adjacency matrix of $G$, denoted by $A(G)$ is the $n \times n$ matrix defined by :

$$
A(G)=\left[a_{i j}\right]_{n \times n} \text { where } a_{i j}= \begin{cases}1 & \text {,if the edge } p_{i} p_{j} \quad \text { in } \mathrm{E}, \\ 0 & , \text { otherwise }\end{cases}
$$

$a_{i j}$ is called the adjacency number of the pair $\left(v_{i}, v_{j}\right)$ of vertices.

Definition: Let the vertex sets of the graphs $G$ and $H$ be $\left\{p_{i}\right.$ $\mid i=1,2, \ldots, m\}$ and $\left\{q_{j} \mid j=1,2, \ldots, n\right\}$ resp.,then the Kronecker product [8], $G \otimes H$,is the graph with vertex set $\left\{\left(p_{i,} q_{j}\right)\right.$ : for $i=1,2, \ldots, m$ and $j=1,2, \ldots, n\}$ such that the adjacency number of the pair $\left(p_{i,} q_{j}\right),\left(p_{k}, q_{\ell}\right)$ is the product of the adjacency numbers of $\left(p_{i}, p_{k}\right)$ in $G$ and $\left(q_{j}, q_{\ell}\right)$ in $H$. $G \otimes H$ is also called direct product(tenser product) of $\mathrm{G}$ and $\mathrm{H}$, and may be denoted by G.H [1]. $G \otimes H$ is also defined as

$\mathrm{V}(G \otimes H)=\mathrm{V}(G) \mathrm{xV}(H)$

$\mathrm{E}(G \otimes H)=\left\{\left(p_{i}, q_{j}\right)\left(p_{k}, q_{\ell}\right) \mid p_{i} p_{k} \in E(G)\right.$ and $\left.q_{j} q_{l} \in E(H)\right\}$.

The Kronecker product is commutative (up to isomorphism) and associative [7] . 
The first important result of the basis number occured in 1937 when MacLane [5] proved that a graph $G$ is planar if and only if $b(G) \leq 2$. In 1981, Schmeichel [6] proved that for $n \geq 5, b\left(K_{n}\right)=3$, and for $m, n \geq 5$, $b\left(K_{m, n}\right)=4$ except for $K_{6,10}, K_{5, n}$ and $K_{6, n}$ in which $n=5,6,7$ and 8 .

Moreover, in 1982, Banks and Schmeichel [2] proved that for $n \geq 7$, $b\left(Q_{n}\right)=4$, where $Q_{n}$ is the n-cube.

The purpose of this paper is to determine upper and lower bounds of the basis number of Kronecker product of a wheel with a path and a cycle.

\subsection{On the Basis Number Of $W_{m} \otimes P_{n}$.}

In this section, we obtain upper and lower bounds for the basis number of kronecker product of a wheel with a path.Let the vertex sets of $C_{m}$ and $P_{n}$ be $Z_{m}$ and $Z_{n}$ respectively, where $Z_{n}$ denotes the additive group of residues modulo n. Let the cycle $C_{m}$ be $0,1,2, \ldots, m-1,0$.

The following lemma is needed in the proof of the following theorem which is due to Weichsel [8].

Lemma1:If $\mathrm{G}$ and $\mathrm{H}$ are connected graphs then the Kronecker product $G \otimes H$ is connected if and only if either $\mathrm{G}$ or $\mathrm{H}$ contains an odd cycle.

Let $W_{m}$ be the join of a cycle $012 \cdots(m-2) 0$ with the vertex $\alpha$ and let $P_{n}=012 \cdots(n-1)$.( See Fig.1).
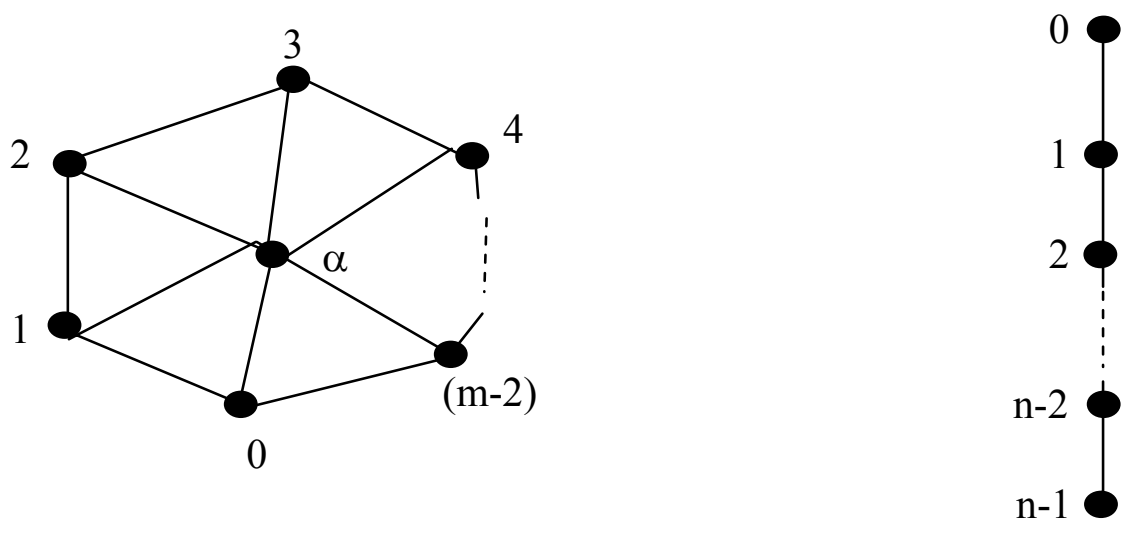

Fig. 1

$$
P_{n}
$$

Theorem 2. For $m \geq 4$ and $n \geq 3,3 \leq b\left(W_{m} \otimes P_{n}\right) \leq 4$.

Proof: One can easily observe from Fig.2, that $W_{m} \otimes P_{3}$ contains a subgraph $\mathrm{H}$ homeomorphic to $K_{3,3}$. Thus by Kuratowskis theorem [3], $W_{m} \otimes P_{3}$ is non planar, since $W_{m} \otimes P_{3}$ is a subgraph of $W_{m} \otimes P_{n}$ for $n \geq 3$; then by MacLanes theorem [5], $b\left(W_{m} \otimes P_{n}\right) \geq 3$. 


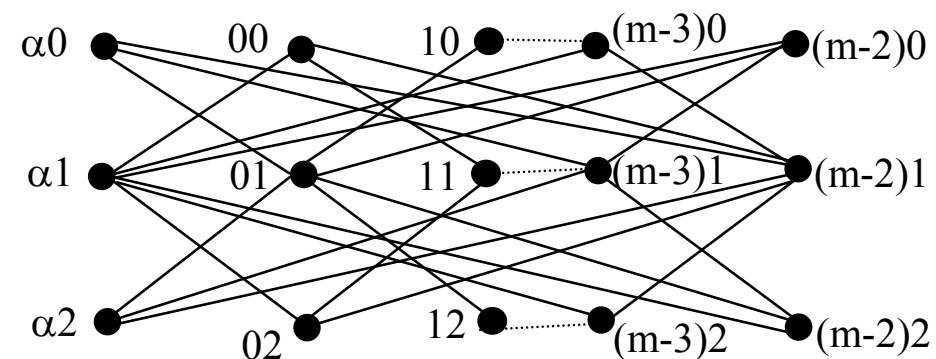

$W_{m} \otimes P_{3}$

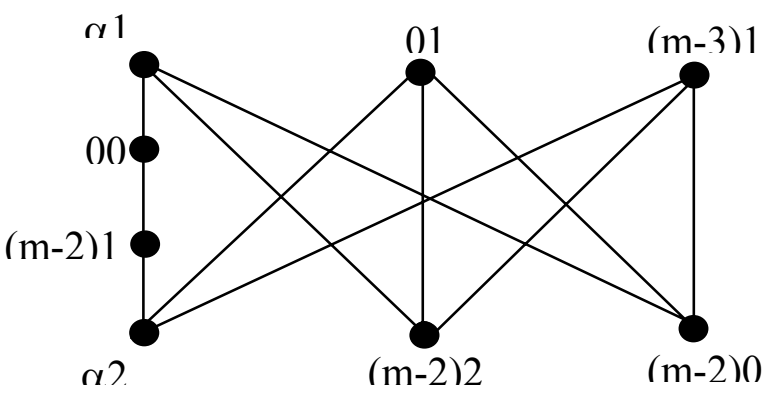

$\mathrm{H}$

Fig.2: $W_{m} \otimes P_{3}$

To complete the proof we find a 4-fold basis $B\left(W_{m} \otimes P_{n}\right)$ for $\xi\left(W_{m} \otimes P_{n}\right)$. We have two cases:

Case (1): “ $m$ ” is even. Let $B=B\left(C_{m-1} \otimes P_{n}\right) \cup N \cup P \cup M,$.

where $B\left(C_{m-1} \otimes P_{n}\right)$ is the basis for $\xi\left(C_{m-1} \otimes P_{n}\right)$ discussed in Theorem 2.2.1[4] in which " $m-1$ " is odd, that is, $B\left(C_{m-1} \otimes P_{n}\right)=\left\{i j,(i-1)(j+1), i(j+2),(i+1)(j+1), i j: i \in Z_{m-1} \quad\right.$ and $\left.\quad j=0,1, \ldots, n-3\right\} \cup\{S\}$, where $S=00,11,20,31, \ldots,(m-2) 0,01,10,21,30, \ldots,(m-2) 1,00$, $N=\{i j,(i+1)(j+1), \alpha j, i(j+1),(i+1) j, \alpha(j+1), i j: i=0,1,2, \ldots, m-3$ and $j=0,1,2, \ldots, n-2\}$, $P=\{(m-2) j, 0(j+1), \alpha j,(m-2)(j+1), 0 j, \alpha(j+1),(m-2) j: j=0,1, \ldots, n-3\}$ and $M=\{\alpha j, i(j+1),(i+1) j,(i+2)(j+1), \alpha j$

and $\alpha(j+1), i j,(i+1)(j+1),(i+2) j, \alpha(j+1): i=0,2,4, \ldots, m-4$ and $j=0,1, \ldots, n-2\}$.

It is clear that

$$
\begin{aligned}
|B| & =(m-1)(n-2)+1+(m-2)(n-1)+(n-2)+(m-2)(n-1) \\
& =3 m n-4 m-4 n+5=\gamma\left(W_{m} \otimes P_{n}\right) .
\end{aligned}
$$

We shall prove that $B$ is independent.

First, the cycles of $N \cup P$ and $M$ are independent for each $j=0,1, \ldots, n-2$ since any linear combination of cycles in $N \cup P$ or $M$ for some $i=0,1, \ldots, m-2$ contains edges of the form, $i j,(i+1)(j+1) \quad$ or $\quad i(j+1),(i+1) j$.

That is, any linear combination of cycles in $N \cup P$ and $M$ is not equal to zero modulo (2). Moreover, for all $j=0,1, \ldots, n-2$, every cycle of $N \cup P$ contains an edge of the form $\alpha j, i(j+1)$ or $\alpha(j+1), i j$ for some 
$i=1,3,5, \ldots, m-2$ which is not present in any cycle of $M$. Also the cycles in $N \cup P \cup M$ satisfy $\left(N_{j} \cup P_{j} \cup M_{j}\right) \cap\left(N_{k} \cup P_{k} \cup M_{k}\right)=\Phi$ for all $j \neq k$ where $N_{j}$ is defined as follows:

It is clear that the vertex set of $W_{m} \otimes P_{n}$ can be partitioned into $V_{0}, V_{1}, \ldots, V_{n-1}$, where

$V_{j}=\{(i, j): i=\alpha, 0,1,2, \ldots, m-2\}$.

Notice that $V\left(W_{m}\right)=\{\alpha, 0,1,2, \ldots, m-2\}$.

Now, $N_{j}$ is the cycle of $N$ that join a vertex of $V_{j}$ to a vertex of $V_{j+1}$, for each $j=0,1, \ldots, n-2$.

By a similar method, we define $P_{j}$ and $M_{j}$.

Moreover, for every nonconsective integers $\mathrm{j}$ and $\mathrm{k}$ in $\{0,1, \ldots, n-2\}$, every cycle in $N_{j} \cup P_{j} \cup M_{j}$ is edge-disjoint with every cycle in $N_{k} \cup P_{k} \cup M_{k}$. Furthermore, if $C_{i}$ is any cycle in $N_{j} \cup P_{j} \cup M_{j}, j=0,1, \ldots, n-3$ then $C_{i}$ contains the edge $i j,(i+1)(j+1)$ which is not contained in any cycle in $N_{j+1} \cup P_{j+1} \cup M_{j+1}$. This shows that $N \cup P \cup M$ is independent. Moreover, the cycles of $N_{j} \cup P_{j} \cup M_{j}$ for all $j=0,1, \ldots, n-2$ are independent from the cycle of $B\left(C_{m-1} \otimes P_{n}\right)$ because if $C_{i}^{\prime}$ is any cycle generated from cycles in $N \cup P \cup M$, then $C_{i}^{\prime}$ contains an edge of the form $\alpha j, i(j+1)$ or $\alpha(j+1), i j$ for some $i=0,1, \ldots, m-2$ which is not present in any cycle of $B\left(C_{m-1} \otimes P_{n}\right)$. Thus $B\left(W_{m} \otimes P_{n}\right)$ is independent set of cycles and so it is a basis for $\xi\left(W_{m} \otimes P_{n}\right)$.

We now consider the fold of $B\left(W_{m} \otimes P_{n}\right)$. Partition, the edge set of $W_{m} \otimes P_{n}$ into $i j,(i+1)(j+1) \quad$ or $\quad i(j+1),(i+1) j$ and $\alpha j, i(j+1)$ or $\alpha(j+1), i j$ in which $i \in Z_{m-1}$ and $j=0,1, \ldots, n-2$. Thus if $e$ is any edge in $W_{m} \otimes P_{n}$ of the form $i j,(i+1)(j+1)$ or $i(j+1),(i+1) j$, then

$$
\begin{array}{lll}
f \quad(e) \leq 2, & f(e) \leq 1, & f(e) \leq 1 \\
B\left(C_{m-1} \otimes P_{n}\right) & N \cup P & M
\end{array}
$$

and so

$$
\begin{aligned}
& f \quad(e) \leq 4 . \\
& B\left(W_{m} \otimes P_{n}\right)
\end{aligned}
$$

While, if $e$ is any edge in $W_{m} \otimes P_{n}$ of the form $\alpha j, i(j+1)$ or $\alpha(j+1), i j$, then

$$
\begin{array}{lll}
f \quad(e)=0, & f(e) \leq 2, & f(e) \leq 2 \\
B\left(C_{m-1} \otimes P_{n}\right) & N \cup P & M
\end{array}
$$

and so 


$$
\begin{aligned}
& f \quad(e) \leq 4 . \\
& B\left(W_{m} \otimes P_{n}\right)
\end{aligned}
$$

Therefore, $B\left(W_{m} \otimes P_{n}\right)$ is a 4-fold basis.

Case (2): “ $m$ ”' is odd. Let $B\left(W_{m} \otimes P_{n}\right)=B^{*}\left(C_{m-1} \otimes P_{n}\right) \cup M^{*} \cup N$.

Where $B^{*}\left(C_{m-1} \otimes P_{n}\right)$ is a basis for $\xi\left(C_{m-1} \otimes P_{n}\right)$ discussed in[1, Theorem 1 , case(2)] namely,

$$
\begin{aligned}
B^{*}\left(C_{m-1} \otimes P_{n}\right)= & \{i j,(i+1)(j-1),(i+2) j,(i+1)(j+1), i j \\
& \left.: i \in Z_{m-1}, j=1,2, \ldots, n-2 \text { and }(j-i) \text { is even }\right\} \cup \\
& \{i j,(i+1)(j-1),(i+2) j,(i+1)(j+1), i j: \\
& \left.i \in Z_{m-1}, j=1,2, \ldots, n-2 \text { and }(j-i) i s \text { odd }\right\},
\end{aligned}
$$

$M^{*}=\{\alpha j, i(j+1),(i+1) j,(i+2)(j+1), \alpha j$ and $\alpha(j+1), i j,(i+1)(j+1),(i+2) j, \alpha(j+1)$

$$
: i=0,2,4, \ldots m-3 \bmod (m-1) \text { and } j=0,1,2, \ldots, n-2\}
$$

and $N$ is same as Case (1).

It is clear that

$$
\begin{aligned}
\left|B\left(W_{m} \otimes P_{n}\right)\right| & =(m-1)(n-2)+(m-1)(n-1)+(m-2)(n-1) \\
& =m n-2 m-n+2+m n-m-n+1+m n-m-2 n+2 \\
& =3 m n-4 m-4 n+5=\gamma\left(W_{m} \otimes P_{n}\right) .
\end{aligned}
$$

As in the proof of Case (1), we can show that $B\left(W_{m} \otimes P_{n}\right)$ is independent set of cycles and so it is a basis for $\xi\left(W_{m} \otimes P_{n}\right)$ of fold 4, that is $B^{*}=B\left(W_{m} \otimes P_{n}\right)$.

Note that if " $m$ " is even and $m \geq 4$, then $W_{m} \otimes P_{2}$ is planar graph,(see Fig.3). 


\section{Ghassan T. Marougi \& Ayhan A. Khalil}

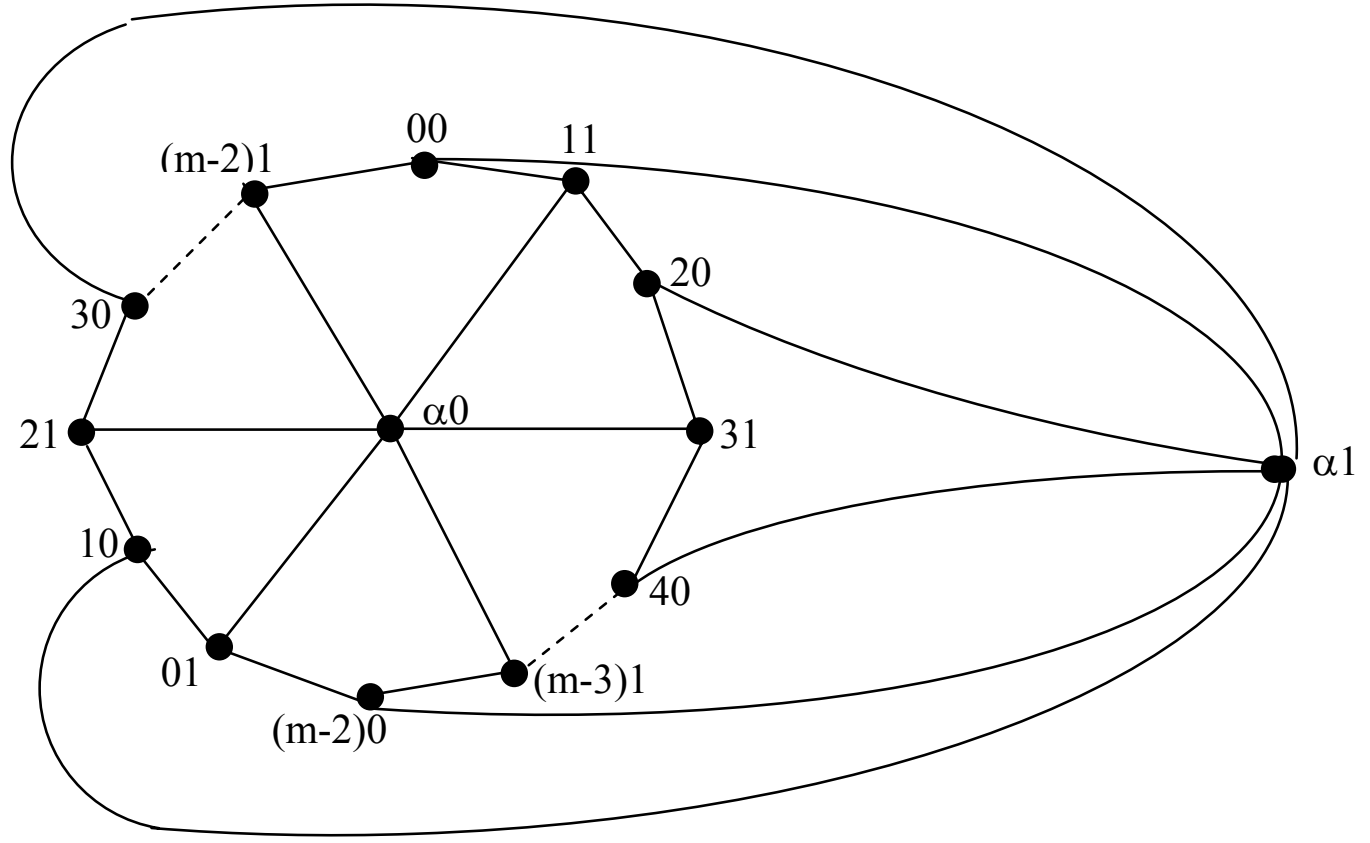

Fig.3

Hence $b\left(W_{m} \otimes P_{2}\right)=2$ for even $m \geq 4$.

While, if " $m$ " is odd and $m \geq 5$, then $W_{m} \otimes P_{2}$ contains a subgraph $K$ homeomorphic to $K_{3,3}$. Therefore by MacLanes theorem [5], the graph $W_{m} \otimes P_{2}$ is nonplanar (see Fig.4). 


\section{UPPER AND LOWER BOUNDS OF THE BASIS...}

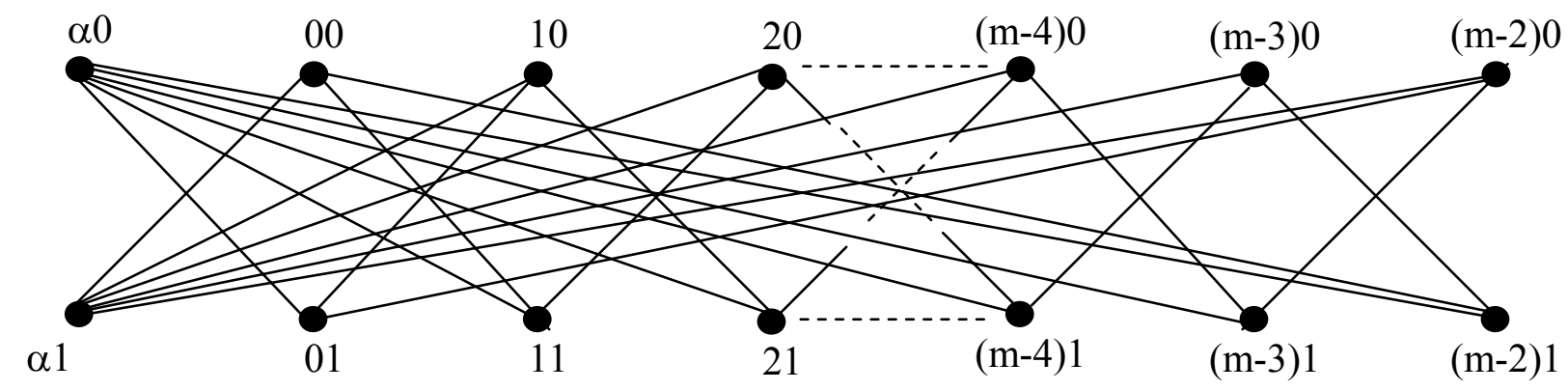

$\mathbf{K}$

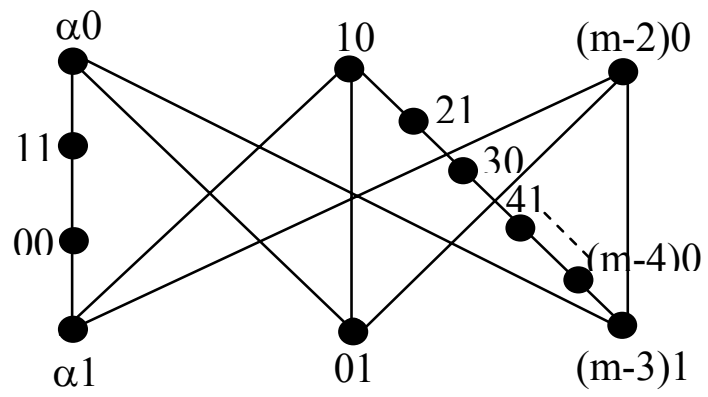

Fig.4: $W_{m} \otimes P_{2}$

Hence $b\left(W_{m} \otimes P_{2}\right)=3$ for even $m \geq 5$.

We conclude the following table

\begin{tabular}{|c||c||c|}
\hline$m$ & $n$ & $b\left(W_{m} \otimes P_{n}\right)$ \\
\hline \hline$m \geq 4, m$ is even & 2 & 2 \\
\hline \hline$m \geq 4, m$ is even & $n \geq 3$ & 3 or 4 \\
\hline \hline$m \geq 5, m$ is odd & 2 & 3 \\
\hline \hline$m \geq 5, m$ is odd & $n \geq 3$ & 3 or 4 \\
\hline
\end{tabular}




\subsection{On the Basis Number Of $W_{m} \otimes C_{n}$.}

In this section, we obtain upper and lower bounds for the basis number of kronecker product of a wheel with a cycle.

Theorem 3. For $m \geq 4, n \geq 3$, we have $3 \leq b\left(W_{m} \otimes C_{n}\right) \leq 5$.

Proof: Since $W_{m} \otimes P_{n}$ is a subgraph of $W_{m} \otimes C_{n}$ for all $m \geq 4$, and $n \geq 3$, then by Theorem 2, we have $W_{m} \otimes C_{n}$ is nonplanar and so by MacLanes theorem [5], we have $b\left(W_{m} \otimes C_{n}\right) \geq 3$.For $m=4$ and $n=3$, ( see Fig.5).

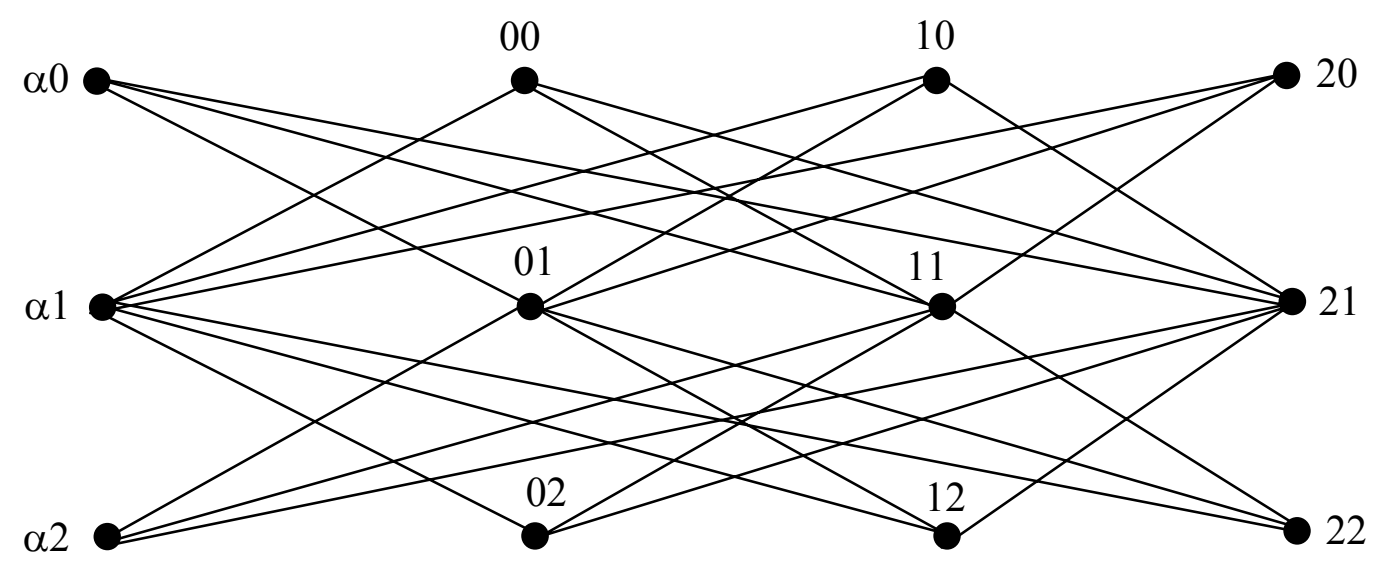

Fig.5: $W_{4} \otimes P_{3}$

To complete the theorem we establish a 5-fold basis $B\left(W_{m} \otimes C_{n}\right)$ for $\xi\left(W_{m} \otimes C_{n}\right)$. We have two possibilities for $m$.

(1) “ $m$ " is even. Then consider the following set of cycles in $W_{m} \otimes C_{n}$ : $B\left(W_{m} \otimes C_{n}\right)=B\left(C_{m-1} \otimes C_{n}\right) \cup N \cup M$.

Where $B\left(C_{m-1} \otimes C_{n}\right)$ is a basis for $\xi\left(C_{m-1} \otimes C_{n}\right)$ discussed in Theorem 2.3.1[4], where " $m-1$ " is odd, that is,

$$
\begin{aligned}
& B\left(C_{m-1} \otimes C_{n}\right)=B\left(C_{m-1} \otimes P_{n}\right) \cup B_{1} \cup\left\{S_{1}, S_{2}\right\}, \text { where } \\
& B\left(C_{m-1} \otimes P_{n}\right)=\left\{i j,(i-1)(j+1), i(j+2),(i+1)(j+1): i \in Z_{m-1} \text { and } j=0,1, \ldots \ldots \ldots, n-3\right\} \cup\{S\}, \\
& S=00,11,20,31, \ldots \ldots \ldots \ldots .,(m-2) 0,01,10,21,30, \ldots \ldots \ldots \ldots,(m-2) 1,00 \\
& B_{1}=\{i j,(i-1)(j+1), i(j+2),(i+1)(j+1), i j: i=0,1, \ldots \ldots \ldots, m-3 \bmod (m-1) \text { and } \\
& \quad j=n-2, n-1(\bmod n)\} \\
& S_{1}=(m-2)(n-2),(m-3)(n-1),(m-2) 0,0(n-1),(m-2)(n-2) \\
& S_{2}=00,(m-2)(n-1),(m-3) 0,(m-4)(n-1), \ldots, 10,0(n-1),(m-2) 0,(m-3)(n-1), \ldots, 20,1(n-1), 00 . \\
& N=\left\{i j,(i+1)(j+1), \alpha j, i(j+1),(i+1) j, \alpha(j+1), i j: i \in Z_{m-1} \text { and } j \in Z_{n}\right\} . \\
& \text { and }
\end{aligned}
$$




$$
\begin{aligned}
M= & \{\alpha j, i(j+1),(i+1) j,(i+2)(j+1), \alpha j \text { and } \alpha(j+1), i j,(i+1)(j+1),(i+2) j, \alpha(j+1) \\
& \left.: i=0,2,4, \ldots ., m-4 \text { and } j \in Z_{n}\right\} .
\end{aligned}
$$

It is clear that

$$
\begin{aligned}
\left|B\left(W_{m} \otimes C_{n}\right)\right| & =m n-n+1+(m-1) n+(m-2) n \\
& =3 m n-4 n+1=\gamma\left(W_{m} \otimes C_{n}\right)
\end{aligned}
$$

We will prove that $B\left(W_{m} \otimes C_{n}\right)$ is independent. It is clear that $N=\bigcup_{j=0}^{n-2}\left(N_{j} \cup P_{j}\right) \cup\left\{N_{n-1}\right\}$ and $M=\bigcup_{j=0}^{n-2}\left(M_{j}\right) \cup\left\{M_{n-1}\right\}$, where $N_{j} \cup P_{j} \cup M_{j}$ for $j=0,1, \ldots, n-2$ are as mentioned in the proof of Theorem 2 . As in the proof of Theorem 2, $N \cup M$ is independent. Moreover for all $i \in Z_{m-1}, N_{n-1} \cup M_{n-1}$ contains the edge $i(n-1),(i+1) 0$, which is not contained in $\bigcup_{j=0}^{n-2}\left(N_{j} \cup P_{j} \cup M_{j}\right)$.

Thus $N \cup M$ is independent set of cycles. Furthermore $N \cup M$ is independent from the cycles of $B\left(C_{m-1} \otimes C_{n}\right)$ since for all $j \in Z_{n}$, if $C_{i}$ is any cycle generated from cycles of $N \cup M$, then $C_{i}$ contains the edge of the from $\alpha j, i(j+1)$ or $\alpha(j+1), i j$ for some $i \in Z_{m-1}$ which is not present in any cycle of $B\left(C_{m-1} \otimes C_{n}\right)$.

Thus $B\left(W_{m} \otimes C_{n}\right)=B\left(C_{m-1} \otimes C_{n}\right) \cup N \cup M$, is independent and so it is a basis. We now consider the fold of $B\left(W_{m} \otimes C_{n}\right)$. Partition the edge-set of $W_{m} \otimes C_{n}$ into $i j,(i+1)(j+1)$ or $i(j+1),(i+1) j$ and $\alpha j, i(j+1)$ or $\alpha(j+1), i j$ for $i \in Z_{m-1}$ and $j \in Z_{n}$. Therefore if $e$ is any edge in $W_{m} \otimes C_{n}$ of the form $i j,(i+1)(j+1)$ or $i(j+1),(i+1) j$, then
$f$
$(e) \leq 3$,
$f(e) \leq 1$,
$f(e) \leq 1$
$B\left(C_{m-1} \otimes C_{n}\right)$
$N$
$M$

and so

$$
\begin{aligned}
& f \quad(e) \leq 5 . \\
& B\left(W_{m} \otimes C_{n}\right)
\end{aligned}
$$

While if $e$ is any edge of the form $\alpha j, i(j+1)$ or $\alpha(j+1), i j$ then

$$
\begin{array}{lll}
f \quad(e)=0, & f(e) \leq 2, & f(e) \leq 2 \\
B\left(C_{m-1} \otimes C_{n}\right) & N & M
\end{array}
$$

and so

$$
\begin{aligned}
& f \quad(e) \leq 4 . \\
& B\left(W_{m} \otimes C_{n}\right)
\end{aligned}
$$

Thus, the basis $B\left(W_{m} \otimes C_{n}\right)$ is of fold 5 . 
(2) " $m$ " is odd, then consider the following set of cycles in $W_{m} \otimes C_{n}$ : $B\left(W_{m} \otimes C_{n}\right)=B^{*}\left(C_{m-1} \otimes P_{n}\right) \cup\left\{F_{i}, F_{i}^{\prime}: i \in Z_{m-1}\right\} \cup N^{*} \cup M^{*}$, where $B^{*}\left(C_{m-1} \otimes P_{n}\right)$ is a basis for $\xi\left(C_{m-1} \otimes P_{n}\right)$ mentioned in [1, Theorem 1, case (2)] and $F_{i}, F_{i}^{\prime}$ are independent cycles [1, Theorem 2, case (1)]. That is, $B^{*}\left(C_{m-1} \otimes P_{n}\right)=\left\{i j,(i+1)(j-1),(i+2) j,(i+1)(j+1), i j: i \in Z_{m-1}, j=1,2, \ldots, n-2\right.$ and $(j-i)$ even $\} \cup\left\{i j,(i+1)(j-1),(i+2) j,(i+1)(j+1), i j: i \in Z_{m-1}\right.$, $j=1,2, \ldots, n-2$ and $(j-i)$ odd $\} \cup\{00,11,20,31, \ldots,(m-3) 0,(m-2) 1,00\}$,

$F_{i}=\{0 i, 1(i-1), 2 i, \ldots,(m-2)(i-1), 0 i\}$, $F_{i}^{\prime}=\{0 i, 1(i+1), 2 i, \ldots,(m-2)(i+1), 0 i\}$, $N^{*}=\left\{i j,(i+1)(j+1), \alpha j, i(j+1),(i+1) j, \alpha(j+1), i j: i=0,1, \ldots, m-3 \quad\right.$ and $\left.\quad j \in Z_{n}\right\}$

and

$$
\begin{aligned}
M^{*}= & \{\alpha j, i(j+1),(i+1) j,(i+2)(j+1), \alpha j \text { and } \alpha(j+1), i j,(i+1)(j+1),(i+2) j, \alpha(j+1) \text { It } \\
& \left.: i=0,2,4, \ldots \ldots . . m-3 \bmod (m-1) \text { and } j \in Z_{n}\right\} .
\end{aligned}
$$

is clear that

$$
\begin{aligned}
\left|B\left(W_{m} \otimes C_{n}\right)\right| & =(m-1)(n-2)+1+2(m-1)+(m-2) n+(m-1) n \\
& =m n-2 m-n+2+1+2 m-2+m n-2 n+m n-n \\
& =3 m n-4 n+1 \\
& =\gamma\left(W_{m} \otimes C_{n}\right) .
\end{aligned}
$$

As in possibility (1), we can prove that $B\left(W_{m} \otimes C_{n}\right)$ is a 5-fold basis for $\xi\left(W_{m} \otimes C_{n}\right)$.

Remark. In contrast to upper bounds of the basis numbers of $W_{m} \otimes P_{n}$ and $W_{m} \otimes C_{n}$ given in Theorem 2 and Theorem 3, one can conjecture that the upper bound for the basis number of kronecker product of two wheels $W_{m}$ and $W_{n}$ is $b\left(W_{m} \otimes W_{n}\right) \leq 10$.

\section{Conjecture:}

(i) What is $b\left(K_{m} \otimes K_{n}\right)$ ? where $W_{m}$ and $W_{n}$ are subgraphs of $K_{m}$ and $K_{n \text {. }}$ resp.

(ii) What did you conjecture about $b\left(G_{1} \otimes G_{2}\right)$ ? 


\section{REFERENCES.}

[1] A.A.Ali, "The basis number of the direct product of paths and cycles", Ars combinatoria, Vol.27 (1989), pp.155-163.

[2] J.A.Banks and E.F.Schmeichel , "The basis number of the n-cube", J.Comb.Theory, Ser.B, Vol.33, No.2, (1982), pp.95-100.

[3] F.Harary, "Graph Theory", 3 rd . printing, Addison-Wesely, Reading, Massachusetts, (1972).

[4] A.A.Khaleel, "On the basis number of Kronecker product of some special graphs",M.Sc.Thesis,Mosul University,(2004).

[5] S.MacLane , "A combinatorial condition for planar graphs", Fund. Math., Vol.28, (1937), pp.22-32.

[6] E.F.Schmeichel , “The basis number of a graph”,J .Comb.Theory, Ser.B, Vol.30,No.2 (1981), pp.123-129.

[7] H.H.Teh, and H.P., Yap, "Some construction problems of homogenous graphs", Bull. Math. Soc., Nanyang Univ., (1964), pp.164-196.

[8] P.M.Weichsel, “The kronecker product of graphs”, Proc,Amer. Math.Soc., Vol.13, (1962), pp.47-52. 